\title{
Thermodynamic Analysis of Magnetohydrodynamic Power Cycle
}

Mamdouh El Haj Assad

Department of Mechanical Engineering, Australian College of Kuwait, Safat-13015, Kuwait

\section{Abstract}

A theoretical model to analyse the performance characteristics of a magnetohydrodynamic generator (MHD) power plant is presented in this work. The internal irreversibilities as well as the external irreversibilities are taken into account in the analysis. General expressions for the power and efficiency are obtained when the MHD generator is operating at constant velocit condition. The results of the power output and the thermal efficiency of the MHD power plant are shown graphically.

\section{Introduction}

Due to the importance of MHD generator power plant in power industry and its competition with gas turbine power plants, MHD power plant is worth studying. In fact MHD power plant is similar to the conventional Brayton cycle except in the latter a gas turbine is used instead of MHD generator. However the source for producing power is completely different.

In MHD power plant, as the working fluid which is a gaseous conductor passes through the generator in which there is a magnetic field, a current is generated. Hence the generator is a direct converter of electricity.

MHD generator is characterized by design simplicity, high operating temperatures as well as possibly high efficiency and absence of rotating parts which cause large centrifugal stresses as the case in gas turbines.

Regenerative Brayton cycle has been investigated with internally reversible [1] and internally irreversible conditions [2]. Recent mathematical models for MHD power plant with partial internal irreversibilities [3] and with variable temperature heat reservoirs [4] have been studied.

The maximum power output of MHD power cycle and the corresponding efficiency without considering the losses in the heat exchanger and in the compressor has been studied [5].

An analysis was carried out to find the maximum power density of MHD power cycle and the corresponding efficiency with considering the loss in the compressor but without considering the loss in the heat exchangers [6].

In the flow systems, thermodynamic irreversibility can be quantified through entropy analysis. Considerable research studies were carried out to examine entropy generation in the flow systems [7]. More research work regarding thermodynamic analysis of MHD an irreversible power plant with different operating conditions and with using regenerator in the power plant can be found in literature [8-11].

The objective of this paper is to investigate the generator effect on the cycle performance for constant velocity operating condition of MHD generator.

\section{Analysis}

A MHD power plant consists of a compressor, heater, MHD generator and cooler. The T-s diagram of MHD power plant is

\section{illustrated in Figure 1.}

The MHD power plant is running between an infinite heat source at temperature $T_{H}$ and infinite heat sink at temperature $T_{L}$. Process 1-2 represents the nonisentropic compression, process 2-3 represents the isobaric heat addition in the hot side heat exchanger from an external heat source, process 3-4 represents the nonisentropic expansion process in the MHD generator, and process 4-1 represents the isobaric heat rejection in the cold side heat exchanger to an external heat sink. The isentropic processes 1-2s along the compressor and 3-4s along the MHD generator are also presented in Figure 1.

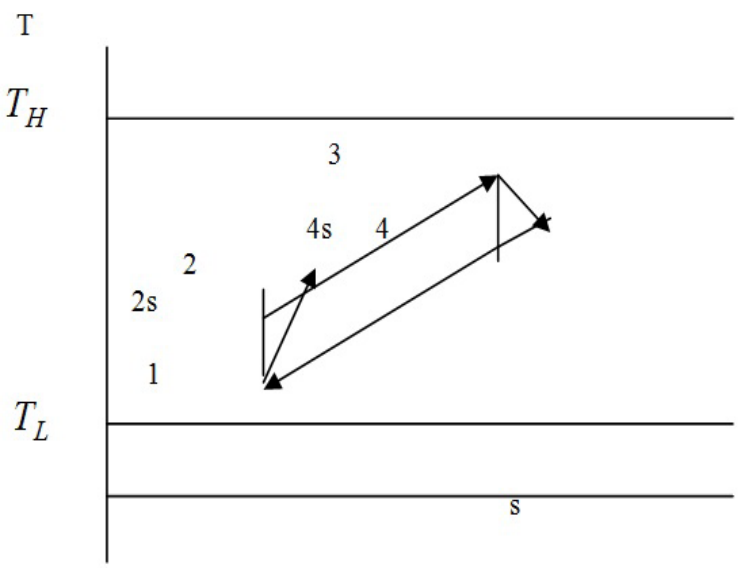

Figure1: T-s diagram of a MHD power plant.

Considering counter flow heat exchangers, the rates of heat addition and heat rejection are, respectively,

$$
Q_{H}=\frac{U_{H} A_{H}\left[\left(T_{H}-T_{2}\right)-\left(T_{H}-T_{3}\right)\right]}{\mathbf{h}\left[\frac{T_{H}-T_{2}}{T_{H}-T_{3}}\right]}=C_{W}\left(T_{3}-T_{2}\right)
$$

"Corresponding Author: Dr. Mamdouh El Haj Assad, Department of Mechanical Engineering, Australian College of Kuwait, Safat-13015, Kuwait; E-mail: m.assad@ack.edu.kw

Citation: Assad MEH (2015) Thermodynamic Analysis of Magnetohydrodynamic Power Cycle. Int J Mech Syst Eng 1: 101. doi: http://dx.doi.org/10.15344/2455$7412 / 2015 / 101$

Copyright: (c) 2015 Assad MEH. This is an open-access article distributed under the terms of the Creative Commons Attribution License, which permits unrestricted use, distribution, and reproduction in any medium, provided the original author and source are credited. 


$$
Q_{L}=\frac{U_{L} A_{L}\left[\left(T_{4}-T_{L}\right)-\left(T_{1}-T_{L}\right)\right]}{\mathbf{h}\left[\frac{T_{4}-T_{L}}{T_{1}-T_{L}}\right]}=C_{W}\left(T_{4}-T_{1}\right)
$$

where $U_{H} A_{H}$ is the heat conductance on the hot side heat exchanger, $U_{L} A_{L}$ heat conductance on the cold side heat exchanger (overall heat transfer coefficient -area product) and $C w$ capacity rate of the working fluid.

From Equations (1) and (2), we obtain

$$
\begin{gathered}
Q_{H}=C_{W} \varepsilon_{H}\left(T_{H}-T_{2}\right) \\
Q_{L}=C_{W} \varepsilon_{L}\left(T_{4}-T_{L}\right)
\end{gathered}
$$

where $\varepsilon_{H}$ and $\varepsilon_{L}$ are, respectively, the effectivenesses of the hot side heat exchanger and cold side heat exchanger, for counter flow heat exchangers, they are given by [12].

$$
\begin{aligned}
& \varepsilon_{H}=1-\exp \left(-N_{H}\right) \\
& \varepsilon_{L}=1-\exp \left(-N_{L}\right)
\end{aligned}
$$

The number of heat transfer units $N_{H}$ and $N_{L}$ are based on minimum heat capacity rates, which are defined

as $N_{H}=\frac{U_{H} A_{H}}{C_{W}}$ and $N_{L}=\frac{U_{L} A_{L}}{C_{W}}$

The power output and efficiency of MHD power plant are, respectively

$$
\begin{aligned}
W & =Q_{H}-Q_{L}=C_{W} \varepsilon_{H}\left(T_{H}-T_{2}\right)-C_{W} \varepsilon_{L}\left(T_{4}-T_{L}\right) \\
\eta & =\frac{W}{Q_{H}}
\end{aligned}
$$

Using Equations (1) and (3), and Equations (2) and (4), the following temperatures can be obtained as

$$
\begin{aligned}
& T_{1}=T_{4}\left(1-\varepsilon_{L}\right)+\varepsilon_{L} T_{L} \\
& T_{3}=T_{2}\left(1-\varepsilon_{H}\right)+\varepsilon_{H} T_{H}
\end{aligned}
$$

The compressor efficiency is defined as

$$
\eta_{c}=\frac{T_{2 s}-T_{1}}{T_{2}-T_{1}}
$$

For the mathematical modelling of MHD power plant, we have assumed that the working fluid is a compressible ideal gas with constant specific heat, and all cycle processes are internally reversible except for the compressor and MHD generator. Using these assumptions and considering one dimensional steady state flow, the momentum and energy equations of the flowing gas in the MHD generator are written as, respectively,

$$
\begin{aligned}
& \rho u \frac{d u}{d z}+\frac{d p}{d z}+J B=0 \\
& \rho u \frac{d}{d z}\left(h+0.5 u^{2}\right)+J E=0
\end{aligned}
$$

where $\rho$ is the gas density, $u$ gas velocity, $h$ gas enthalpy, $p$ gas pressure , $J$ current density, $B$ magnetic field and $E$ electric field.
The electrical efficiency of MHD generator is defined as [13]

$$
\eta_{e}=\frac{J E}{u J B}
$$

Equations (11) and (14) give a clear idea about the internal irreversibilities of the compressor and generator, respectively. As the efficiencies of the compressor and generator approach $100 \%$, the MHD power plant is internally reversible but still externally reversible due to the finite heat transfer between the power plant and its thermal surroundings.

\section{Constant velocity}

In this case the flowing gas in the generator does not slow down but the electrical power comes from the work done by the expansion of the gases against the magnetic forces, i.e. from the enthalpy or thermal energy rather than the kinetic energy of gases.

Using Equations (12) - (14) gives

$\left(\frac{k-1}{k}\right) \eta_{e} \frac{d p}{p}=\frac{d T}{T}$

where $k$ is the specific heat ratio of the working fluid.

Equation (15) can be integrated for the process 3-4 to give

$\frac{T_{3}}{T_{4}}=X^{\eta_{e}}$

where the temperature ratio $X$ is

$$
X=\frac{T_{2 s}}{T_{1}}=\left(\frac{p_{2}}{p_{1}}\right)^{(k-1) / k}
$$

By using Equations (7), (9) - (11) and (15) - (17), the power output $W$ can be written in terms of $X$ only, and defining a dimensionless power output as $W_{U}^{*}=\frac{W}{C_{W} T_{L}}$, we obtain

$$
W_{U}^{*}=\frac{a X^{\eta_{e}+1}+\left(b \eta_{c}-a\right) X^{\eta_{e}}+c X-b \eta_{c}-c}{\eta_{c} X^{\eta_{e}}+d X+d\left(\eta_{c}-1\right)}
$$

The efficiency for constant gas velocity case can be expressed as

$$
\eta_{U}=\frac{a X^{\eta_{e}+1}+\left(b \eta_{c}-a\right) X^{\eta_{e}}+c X-b \eta_{c}-c}{a X^{\eta_{e}+1}+\left[\eta_{c}(a+e)-a\right] X^{\eta_{e}}+(f-e) X+(f-e)\left(\eta_{c}-1\right)}
$$

where

$$
\begin{aligned}
& a=-\varepsilon_{L} \varepsilon_{H} \\
& b=\gamma \varepsilon_{H}-\varepsilon_{L} \varepsilon_{H}+\varepsilon_{L} \\
& c=-\gamma \varepsilon_{H}\left(1-\varepsilon_{L}\right)+\varepsilon_{L} \varepsilon_{H}-\varepsilon_{L} \\
& d=-\left(1-\varepsilon_{L}\right)\left(1-\varepsilon_{H}\right) \\
& e=\gamma \varepsilon_{H}
\end{aligned}
$$

$$
f=\gamma \varepsilon_{L} \varepsilon_{H}
$$


where $\gamma$ is defined as $\gamma=\frac{T_{H}}{T_{L}}$

\section{Efficiency at maximum power}

The maximum dimensionless power outputs for constant velocity and constant Mach number can be found by maximizing Equations (18) with respect to $X$ as

$$
\frac{d W_{U}^{*}}{d X}=0
$$

The solution of Equation (21) is too complex to obtain a simple analytical solution for the power output, hence a numerical solution is given in the next section.

For $\eta_{e}=\eta_{c}=1$ and $\varepsilon_{H}=\varepsilon_{L}$, Equation (21) gives . For this special case the efficiency of MHD power plant at maximum power output is obtained as

$$
\eta=1-\left(\frac{T_{L}}{T_{H}}\right)^{1 / 2}
$$

which is known as Curzon and Ahlborn efficiency.

\section{Results and Discussion}

In this section, we present the results for constant velocity MHD generator for $k=1.4, N_{H}=N_{L}=1$ and $\gamma=\frac{T_{H}}{T_{L}}=5$

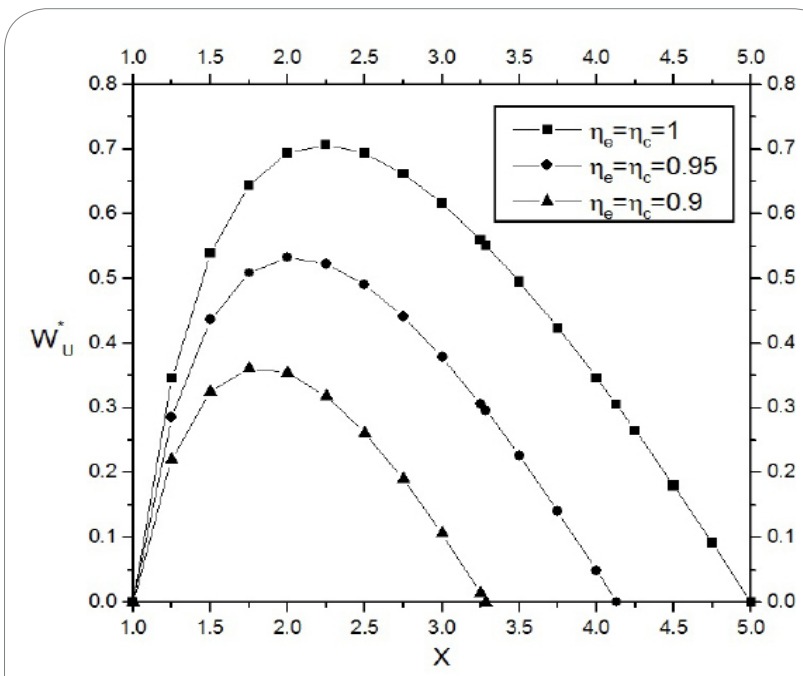

Figure 2: Variation of $\mathrm{X}$ with $\mathrm{w}_{\mathrm{U}}^{*}$.

Figure 3 shows the variation of $X$ with $\eta_{U}$. For $\eta_{e}=\eta_{c}=1$

(endoreversible cycle), the efficiency of MHD power plant increases monotonically with $X$, however for $\eta_{e}<1$ and $\eta_{c}<1$, there exists a maximum. As can be seen from Figures 2 and 3, the maximum efficiency of MHD power plant occurs at higher X values than that of the maximum dimensionless power output.

One of the important points that worth mentioning is for $\eta_{e}=\eta_{c}=1$ ,the working fluid has zero electrical resistivity and the constant velocity case is internally reversible by which it becomes identical to Joule-Brayton cycle. The results show that for $\eta_{e}=\eta_{c}=1$, the cycle efficiency reached Carnot efficiency at $X=5$, for which the power output is zero, hence the cycle is internally and externally reversible, i.e. the heat engine is running at zero speed however the efficiency at
$X=1$, is zero and hence zero power output, i.e. the heat engine is running at infinite speed.

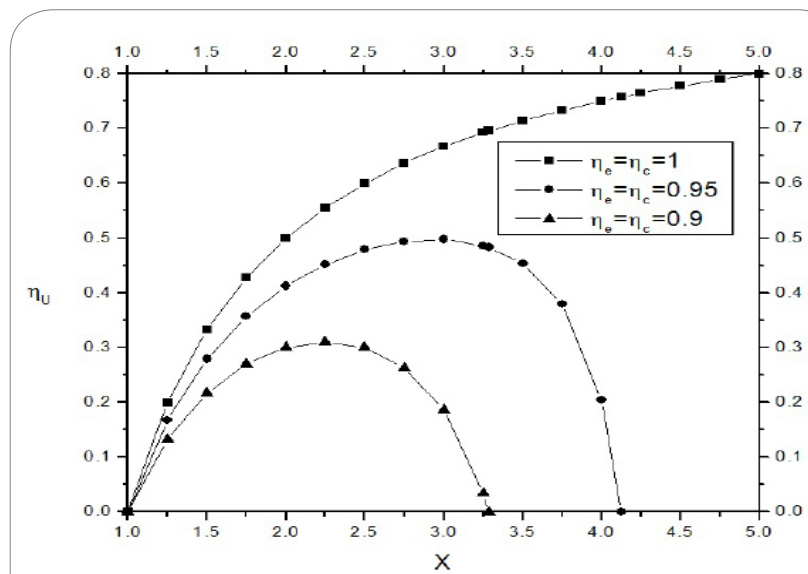

Figure 3: Variation of $\mathrm{X}$ with $\eta_{\mathrm{U}}$.

\section{Conclusions}

An irreversible MHD power plant operating between an infinite heat source and heat sink has been analysed by using finite-time thermodynamics. The non-isentropic processes in the compressor and generator have been taken into account by introducing the compressor and generator efficiencies. The non-isentropic processes lower the power output as well as efficiency when compared with the endoreversible MHD power plant under the same operating conditions.

\section{Competing Interests}

The authors declare that they have no competing interests exits.

\section{References}

1. Cheng $\mathrm{CH}$, Chen CK (1996) Power Optimization of Endoreversible Regenerative Brayton Cycle. Energy 21: 241-247.

2. Wu C, Chen L, Sun F (1996) Performance of a Regenerative Brayton Heat Engine. Energy 21: 71-76.

3. El Haj Assad M, Wu C (1999) Finite Time Thermodynamic Analysis of a MHD Power Plant, Recent Advances in Finite - Time Thermodynamics, Eds. Chih Wu et al., Nova Science Publishers 239-249.

4. Chen L, Grong J, Sun F, Wu C (2002) Heat Transfer Effect on the Performance of MHD Power Plant. Energy Conversion and Management 43: 2085-2095.

5. Aydin M, Yavuz H (1993) Application of Finite-Time Thermodynamics to MHD Power Cycles. Energy 18: 907-911.

6. Sahin B, Kodal A, Yavuz H (1996) A Performance Analysis of MHD Power Cycle Operating at Maximum Power Density. J Physics D: Applied Physics 29: 1473-1475.

7. Bejan A (1995) Entropy Generation Minimization. CRC Press: New York.

8. El Haj Assad M (2000) Thermodynamic Analysis of an Irreversible MHD Power Plant. Int J Energy Research 24.

9. El Haj Assad M (2004) Performance of a Regenerative MHD Power Plant. Int J Energy and Power Systems 24.

10. El Haj Assad M (2007) Optimum Performance of an Irreversible MHD Power Plant. Int J Exergy 4.

11. El Haj Assad M, Wu C (2008) Thermodynamic Performance of an Irreversible MHD Power Cycle Running at Constant Mach Number. Int J Ambient Energy 29

12. Kays W, London MAL (1965) Compact Heat Exchangers, McGraw-Hill: New York.

13. Spring KH (1965) Direct Generation of Electricity. Academic Press: New York. 